\title{
HITCHIN SYSTEMS AT LOW GENERA
}

\author{
KRZYSZTOF GAWȨDZKI \\ I.H.E.S., C.N.R.S., F-91440 Bures-sur-Yvette, France \\ PASCAL TRAN-NGOC-BICH \\ Université de Paris-Sud, F-91405 Orsay, France
}

\begin{abstract}
The paper gives a quick account of the simplest cases of the Hitchin integrable systems and of the Knizhnik-Zamolodchikov-Bernard connection at genus 0, 1 and 2. In particular, we construct the action-angle variables of the genus 2 Hitchin system with group $S L_{2}$ by exploiting its relation to the classical Neumann integrable systems.
\end{abstract}

\section{Hitchin systems}

As was realized by Hitchin in [16], a large family of integrable systems may be obtained by a symplectic reduction of a chiral 2-dimensional gauge theory. Let $\Sigma$ denote a closed Riemann surface of genus $g$ and let $G$ be a complex Lie group which we shall assume simple, connected and simply connected. We shall denote by $\mathcal{A}$ the space of $\operatorname{Lie}(G)$-valued 0,1-gauge fields $A=A_{\bar{z}} d \bar{z}$ on $\Sigma$. Hitchin's construction 16] associates to $\Sigma$ and $G$ an integrable system obtained by a symplectic reduction of the infinite-dimensional complex symplectic manifold $T^{*} \mathcal{A}$ of pairs $(A, \Phi)$ where $\Phi=\Phi_{z} d z$ is a $\operatorname{Lie}(G)$-valued 1,0-Higgs field. The holomorphic symplectic form on $T^{*} \mathcal{A}$ is

$$
\int_{\Sigma} \operatorname{tr} \delta \Phi \delta A
$$

where tr stands for the Killing form on $\operatorname{Lie}(G)$ normalized so that $\operatorname{tr} \phi^{2}=2$ for the long roots $\phi$. The local gauge transformations $h \in \mathcal{G} \equiv \operatorname{Map}(\Sigma, G)$ act on $T^{*} \mathcal{A}$ by

$$
A \longmapsto{ }^{h} A \equiv h A h^{-1}+h \bar{\partial} h^{-1}, \quad \Phi \longmapsto{ }^{h} \Phi \equiv h \Phi h^{-1}
$$

preserving the symplectic form. The corresponding moment map $\mu: T^{*} \mathcal{A} \longrightarrow \operatorname{Lie}(\mathcal{G})^{*} \cong$ $\wedge^{2}(\Sigma) \otimes \operatorname{Lie}(G)$ takes the form

$$
\mu(A, \Phi)=\bar{\partial} \Phi+A \Phi+\Phi A .
$$

The symplectic reduction gives the reduced phase space

$$
\mathcal{P}=\mu^{-1}(\{0\}) / \mathcal{G}
$$

\footnotetext{
${ }^{1}$ one may work in a fixed smoothness class and use the Sobolev norms to define topology in $\mathcal{A}$
} 
with the symplectic structure induced from that of $T^{*} \mathcal{A}$. $\mathcal{P}$ may be identified with the complex cotangent bundle $T^{*} \mathcal{N}$ to the orbit space $\mathcal{N}=\mathcal{A} / \mathcal{G}$ and $\mathcal{N}$, in turn, with the moduli space of holomorphic $G$-bundles on $\Sigma$. More precisely, care should be taken to avoid non-generic bad orbits in order to obtain tractable orbit spaces. This may be done by considering only gauge fields $A$ leading to stable $G$-bundles forming smooth moduli space $\mathcal{N}_{s}$ or those leading to semi-stable bundles giving rise to a, generally singular, compactification $\mathcal{N}_{s s}$ of $\mathcal{N}_{s}$. In what follows we shall be somewhat cavalier about such details.

The Hitchin system has $\mathcal{P}$ as its phase space. Its Hamiltonians are obtained the following way. Let $p$ be a homogeneous $A d$-invariant polynomial on $\operatorname{Lie}(G)$ of degree $d_{p}$. Then

$$
h_{p}(A, \Phi)=p(\Phi)=p\left(\Phi_{z}\right)(d z)^{d_{p}}
$$

defines a $d_{p}$-differential on $\Sigma$ which is holomorphic if $\mu(A, \Phi)=0$. Since $h_{p}$ is constant on the orbits of $\mathcal{G}$, it descends to the reduced phase space:

$$
h_{p}: \mathcal{P} \longrightarrow H^{0}\left(K^{d_{p}}\right) .
$$

Here $K$ stands for the canonical bundle (of covectors $\propto d z)$ and $H^{0}\left(K^{d_{p}}\right)$ is the (finite-

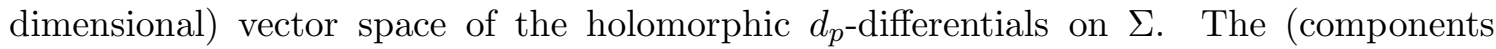
of) $h_{p}$ Poisson-commute (they Poisson-commute already as functions on $T^{*} \mathcal{A}$ since they depend only on the "momenta" $\Phi)$. The point of Hitchin's construction is that, by taking a complete system of polynomials $p$, one obtains on $\mathcal{P}$ a complete system of Hamiltonians in involution. For the matrix groups, the values of Hamiltoniens $h_{p}$ at a point of $\mathcal{P}$ may be encoded in the spectral curve $\mathcal{C}$ obtained by solving the characteristic equation

$$
\operatorname{det}(\Phi-\xi)=0
$$

for $\xi \in K$. The spectral curve of the eigenvalues $\xi$ is a ramified cover of $\Sigma$. The corresponding eigenspaces of $\Phi$ form then a holomorphic line bundle over $\mathcal{C}$ belonging to a subspace of the Jacobian of $\mathcal{C}$ on which the Hamiltonians $h_{p}$ induce linear flows.

For the quadratic polynomial $p_{2}=\frac{1}{2} \operatorname{tr}$, the map $h_{p_{2}}$ takes values in the space of holomorphic quadratic differentials $H^{0}\left(K^{2}\right)$. This is the space cotangent to the moduli space of complex curves $\Sigma$. Variations of the complex structure of $\Sigma$ are described by Beltrami differentials $\delta \mu=\delta \mu_{\bar{z}}^{z} \partial_{z} d \bar{z}$ such that $z^{\prime}=z+\delta z$ with $\partial_{\bar{z}} \delta z=\delta \mu_{\bar{z}}^{z}$ gives new complex coordinates. The Beltrami differentials $\delta \mu$ may be paired with quadratic differentials $\beta$ by

$$
(\beta, \delta \mu) \mapsto \int_{\Sigma} \beta \delta \mu
$$

The differentials $\delta \mu=\bar{\partial}(\delta \xi)$, where $\delta \xi$ is a vector field on $\Sigma$, describe variations of the complex structure due to diffeomorphisms of $\Sigma$ and they pair to zero with $\beta$. The quotient space $H^{1}\left(K^{-1}\right)$ of differentials $\delta \mu$ modulo $\bar{\partial}(\delta \xi)$ is the tangent space to the moduli space of curves $\Sigma$ and $H^{0}\left(K^{2}\right)$ is its dual. The pairing (1.8) defines then for each $[\delta \mu] \in H^{1}\left(K^{-1}\right)$ a Hamiltonian

$$
h_{\delta \mu} \equiv \int_{\Sigma} h_{p_{2}} \delta \mu
$$


The Hamiltonians $h_{\delta \mu}$ Poisson-commute for different $\delta \mu$.

Hitchin's construction possesses a natural generalization [20] [22][5] [8]. Let $x_{n} \in \Sigma$ be a finite family of distinct points in $\Sigma$ and $\mathcal{O}_{n}$ a family of (co)adjoint orbits in $\operatorname{Lie}(G)^{*} \cong$ $\operatorname{Lie}(G)$.

$$
\mathcal{O}=\left\{\sum_{n} \lambda_{n} \delta_{x_{n}} \mid \lambda_{x} \in \mathcal{O}_{n}\right\}
$$

where $\delta_{x}$ stands for the Dirac delta measure at $x$, forms a coadjoint orbit of the group $\mathcal{G}$ of local gauge transformations. In the symplectic reduction we may replace definition (1.4) with

$$
\mathcal{P}_{\mathcal{O}}=\mu^{-1}(\{\mathcal{O}\}) / \mathcal{G} \cong \mu^{-1}\left(\sum \lambda_{n} \delta_{x_{n}}\right) / \mathcal{G}_{\underline{\lambda}, \underline{x}}
$$

where $\mathcal{G}_{\underline{\lambda}}, \underline{x}$ is the subgroup of $\mathcal{G}$ fixing $\sum \lambda_{n} \delta_{x_{n}}$. Upon restriction to properly defined stable pairs $(A, \Phi), \mathcal{P}_{\mathcal{O}}$ gives a smooth space with a semi-stable compactification [20]. Its second representation in (1.11) allows to equip $\mathcal{P}_{\mathcal{O}}$ with the symplectic structure inherited from $T^{*} \mathcal{A}$. The reduced Hamiltonians $h_{p}$ take now values in $H^{0}\left(K^{d_{p}}\left(d_{p} \sum x_{n}\right)\right)$, i.e. define meromorphic $d_{p}$-differentials with possible poles at $x_{n}$ of order $\leq d_{p}$ and they still define an integrable system on $\mathcal{P}_{\mathcal{O}}$. In particular, $h_{p_{2}}$ takes values in $H^{0}\left(K^{2}\left(2 \sum x_{n}\right)\right)$ which is dual to $H^{1}\left(K^{-1}\left(-2 \sum x_{n}\right)\right)$, the tangent space to the moduli space of curves $\Sigma$ with fixed punctures $x_{n}$ and first jets at $x_{n}$ of holomorphic local parameters $z_{n}, z_{n}\left(x_{n}\right)=0$. The corresponding Beltrami differentials $\delta \mu$ behave like $\mathcal{O}\left(z_{n}^{2}\right)$ around $x_{n}$ and they are taken modulo $\bar{\partial}(\delta \xi)$ where the vector fields $\xi$ are also $\mathcal{O}\left(z_{n}^{2}\right)$ around $x_{n}$ (such vector fields do not change the first jets at $x_{n}$ of the local parameters $z_{n}$ ). $\delta \mu$ may still be coupled to quadratic differentials $\beta \in H^{0}\left(K^{2}\left(2 \sum x_{n}\right)\right)$ by (1.8) and Eq. (1.9) defines for $[\delta \mu] \epsilon$ $H^{1}\left(K^{-1}\left(-2 \sum x_{n}\right)\right)$ Hamiltonians on $\mathcal{P}_{\mathcal{O}}$ that are in involution.

\section{Knizhnik-Zamolodchikov-Bernard connection}

The phase space $\mathcal{P} \cong T^{*} \mathcal{N}$ may be (geometrically) quantized by considering the space $H^{0}\left(\mathcal{L}^{k}\right)$ of holomorphic sections of the $k^{\text {th }}$ power of the determinant line bundle $\mathcal{L}$ over $\mathcal{N}$ (more exactly, over its semi-stable version $\mathcal{N}_{s s}$ ) as the space of quantum states. Such sections are given by holomorphic functions $\psi$ on $\mathcal{A}$ satisfying the Ward identity

$$
\psi(A)=\mathrm{e}^{-k S(h, A)} \psi\left({ }^{-1} A\right)
$$

for $h \in \mathcal{G}$ and with $S(h, A)$ standing for the action of the gauged Wess-Zumino-NovikovWitten (WZNW) model. The identity (2.1) expresses the gauge invariance on the quantum level. The vector spaces $H^{0}\left(\mathcal{L}^{k}\right)$ arise naturally in the context of the WZNW model and of the Chern-Simons theory [25]. They are finite-dimensional and their dimension is given by the Verlinde formula [24]. Put together for different complex structures of $\Sigma$, they form a holomorphic vector bundle $\mathcal{W}$ over the moduli space of complex curves. In the language of functions $\psi$, the $\bar{\partial}$-operator of this bundle is given by

$$
\bar{\partial} \overline{\delta \mu} \psi=\left(d \overline{\delta \mu}+\frac{k}{4 \pi i} \int_{\Sigma} \operatorname{tr}(A \overline{\delta \mu}) A\right) \psi
$$


where $d \overline{\delta \mu}$ differentiates $\psi$ viewed as a function of the unitary gauge field $B=-A^{*}+A=$ $-A_{\bar{z}}^{*} d z+A_{\bar{z}} d \bar{z}$ (functions of $B$ are naturally identified for different complex structures on $\Sigma$ ). The bundle $\mathcal{W}$ may be equipped with a projectively flat connection $\nabla^{\mathrm{KZB}}$ [25] 117] which may be traced back to the works of Knizhnik-Zamolodchikov [18] and Bernard [3] [4]. In the present description of $\mathcal{W}$, the KZB connection takes the form [12]

$$
\begin{aligned}
\nabla_{\delta \mu}^{\mathrm{KZB}} \psi & =\left(d_{\delta \mu}-\int_{\Sigma} \operatorname{tr} A^{*}\left(\frac{\delta}{\delta A} \delta \mu\right)-\frac{\pi i}{\kappa} \int_{\Sigma} \operatorname{tr} \bullet \frac{\delta}{\delta A}\left(\frac{\delta}{\delta A} \delta \mu\right)\right. \\
\nabla \frac{\mathrm{KZB}}{\delta \mu} \psi & =\bar{\partial}_{\overline{\delta \mu}} \psi
\end{aligned}
$$

where $\kappa=k+g^{\vee}$ with $g^{\vee}$ denoting the dual Coxeter number of $G$. The symbol $\bullet:$ indicates that one should remove the singularity at the coinciding points of $\frac{\delta}{\delta A(x)} \frac{\delta}{\delta A(y)} \psi$ before setting $x=y$. How this is precisely done depends on some choices (e.g. of a projective connection or a metric on each $\Sigma$ ) but the choices lead to connections differing by addition of a scalar form.

The second order operator on the right hand side of Eq. (2.3) has the principal symbol (obtained by replacement of $\frac{\delta}{\delta A}$ by $\frac{k}{2 \pi i} \Phi$ ) proportional to the Hitchin Hamiltonian $h_{\delta \mu}$. The KZB connection $\nabla_{\delta \mu}^{\mathrm{KZB}}$ may be considered a quantization of $\frac{k}{2 \pi i} h_{\delta \mu}$ which, instead of acting in a fixed space $H^{0}\left(\mathcal{L}^{k}\right)$ relates two such spaces for the complex structures differing by $\delta \mu$ [17]. Note that if we rescale $\delta \mu \mapsto \kappa \delta \mu$, we should obtain from the KZB connection in the limit $\kappa \rightarrow 0$ operators acting in the space $H^{0}\left(\mathcal{L}^{-g^{\vee}}\right)$ corresponding to a fixed complex structure. This space becomes non-trivial if we admit singular sections of $\mathcal{L}$ or work with higher cohomologies of $\mathcal{L}^{-g^{\vee}}$. It also admits a quantization of the non-quadratic Hitchin Hamiltonians [2] [11]. For $k \neq-g^{\vee}$ we may also obtain from Eq. (2.3) operators in a single space if we chose a local trivialization of the bundle $\mathcal{W}$ (or of a bundle $\mathcal{W}^{\prime} \supset \mathcal{W}$ ).

The above quantization extends to the case of the phase space $\mathcal{P}_{\mathcal{O}}$ if the coadjoint orbits $\mathcal{O}_{n}$ associated to points $x_{n} \in \Sigma$ correspond to irreducible holomorphic representations of $G$ in vector spaces $V_{n}$ (i.e. to irreducible unitary representations of the compact form of $G)$. The quantum states are now represented by holomorphic maps on $\mathcal{A}$ with values in $V=\underset{n}{\otimes} V_{n}$ satisfying the Ward identities

$$
\psi(A)=\mathrm{e}^{-k S(h, A)} \underset{n}{\otimes} h\left(x_{n}\right) \psi\left(h^{-1} A\right)
$$

for $h \in \mathcal{G}$ generalizing Eq. (2.1). The spaces of solutions are still finite-dimensional and form a holomorphic vector bundle over the moduli space of punctured curves with first jets of local parameters at the punctures. The complex structure and the KZB connection are given by the same formulae with Beltrami differentials $\delta \mu$ restricted to behave like $\mathcal{O}\left(z_{n}^{2}\right)$ at the punctures. Since $\frac{\delta}{\delta A(x)} \psi=\mathcal{O}\left(z_{n}^{-1}\right)$ and $\operatorname{tr} \bullet \frac{\delta}{\delta A(x)} \frac{\delta}{\delta A(x)} \bullet \psi=\mathcal{O}\left(z_{n}^{-2}\right)$ around $x_{n}$, there is no problem of convergence of the integrals over $\Sigma$. Again, $\nabla_{\delta \mu}^{\mathrm{KZB}}$ may be viewed as the quantization of the Hitchin Hamiltonian $\frac{k}{2 \pi i} h_{\delta \mu}$ and all the above remarks apply. 


\section{Genus zero}

Up to diffeomorphisms, there is only one Riemann surface of genus zero: the Riemann sphere $\mathbb{P}^{1}=\mathbb{C} \cup\{\infty\}$. On $\mathbb{P}^{1}$, the gauge orbit of the zero gauge field is open and dense in $\mathcal{A}$, i.e. the generic gauge field takes the form

$$
A=h^{-1} \bar{\partial} h
$$

where $h \in \mathcal{G}$ is determined up to left multiplication by a constant $g \in G$. The equation $\mu(A, \Phi)=\sum_{n} \lambda_{n} \delta_{z_{n}}$, with $\lambda_{n}$ belonging to the (co)adjoint orbit $\mathcal{O}_{n}$ associated to the puncture $z_{n}$, becomes

$$
\bar{\partial}\left({ }^{h} \Phi\right)=\sum_{n} \nu_{n} \delta_{z_{n}}
$$

where $\nu_{n}=h\left(z_{n}\right) \lambda_{n} h\left(z_{n}\right)^{-1} \in \mathcal{O}_{n}$. This equation has a (unique) solution

$$
{ }^{h} \Phi(z)=\sum_{n} \frac{\nu_{n}}{z-z_{n}} \frac{d z}{2 \pi i}
$$

if and only if the sum of residues is zero, i.e. if $\sum_{n} \nu_{n}=0$. We obtain then for $\mathcal{P}_{\mathcal{O}}$ defined by Eq. (1.11):

$$
\mathcal{P}_{\mathcal{O}} \cong\left\{\underline{\nu} \in \underset{n}{\times} \mathcal{O}_{n} \mid \sum_{n} \nu_{n}=0\right\} / G
$$

The (co)adjoint orbits carry a natural symplectic structure leading to the Poisson bracket $\left\{\nu^{a}, \nu^{b}\right\}=i f^{a b c} \nu^{b}$ for $\nu^{a}=\operatorname{tr} t^{a} \nu$ where $t^{a}$ are the generators of $\operatorname{Lie}(G)$ s.t. $\operatorname{tr} t^{a} t^{b}=\delta^{a b}$ and $\left[t^{a}, t^{b}\right]=i f^{a b c} t^{c}$. It is easy to check that the complex symplectic structure on $\mathcal{P}_{\mathcal{O}}$ coincides with the one obtained by the symplectic reduction of $\times_{n} \mathcal{O}_{n}$ with respect to the diagonal action of $G$.

The Hamiltonians $h_{p}$ are

$$
h_{p}(z)=p\left(\sum_{n} \frac{\nu_{n}}{z-z_{n}}\right)\left(\frac{d z}{2 \pi i}\right)^{d_{p}} .
$$

In the special case $p_{2}=\frac{1}{2} \operatorname{tr}$,

$$
h_{p_{2}}=\frac{1}{2} \sum_{n, m} \frac{\nu_{n}^{a} \nu_{m}^{a}}{\left(z-z_{n}\right)\left(z-z_{m}\right)}\left(\frac{d z}{2 \pi i}\right)^{2}=\sum_{n}\left(\frac{1}{\left(z-z_{n}\right)^{2}} \delta_{n}+\frac{1}{z-z_{n}} h_{n}\right)\left(\frac{d z}{2 \pi i}\right)^{2}
$$

where

$$
\delta_{n}=\frac{1}{2} \nu_{n}^{a} \nu_{n}^{a}, \quad h_{n}=\sum_{m \neq n} \frac{\nu_{n}^{a} \nu_{m}^{a}}{z_{n}-z_{m}} .
$$

Let $\delta \mu$ be a Beltrami differential regular at infinity and behaving like $\mathcal{O}\left(\left(z-z_{n}\right)^{2}\right)$ around the insertions. Necessarily, $\delta \mu=\bar{\partial}(\delta \xi)$ for a regular vector field $\delta \xi=\delta \xi^{z} \partial_{z}$ on $\mathbb{P}^{1}$. $\delta \xi$ is determined up to infinitesimal Möbius transformations $\left(a+b z+c z^{2}\right) \partial_{z}$. We may take $z^{\prime}=z+\delta \xi^{z}$ as the new complex coordinate on $\mathbb{P}^{1}$ with the modified complex structure. 
The modification is then equivalent to the shift $\delta z_{n}=\delta \xi^{z}\left(z_{n}\right)$ of the insertion points and the shift $\delta \chi_{n}=\chi_{n} \partial_{z}\left(\delta \xi_{n}^{z}\right)\left(z_{n}\right)$ of the first jet of the local parameter at the punctures parametrized by the $\partial_{z}$-derivative $\chi_{n}$ of the parameter at $z_{n}$. An easy calculation involving cutting out small balls around the insertions and integration by parts shows that

$$
\begin{array}{r}
h_{\delta \mu}=\sum_{n} \int_{\Sigma}\left(\frac{1}{\left(z-z_{n}\right)^{2}} \delta_{n}+\frac{1}{z-z_{n}} h_{n}\right)\left(\frac{d z}{2 \pi i}\right)^{2} \bar{\partial}(\delta \xi) \\
=\frac{1}{2 \pi i} \sum_{n}\left(\delta_{n} \chi_{n}^{-1} \delta \chi_{n}+h_{n} \delta z_{n}\right) .
\end{array}
$$

The quantum states $\psi$ at genus zero may be labeled by their values $\psi(0)$ at $A=0$ which belong to the subspace $V^{G}$ of the $G$-invariant tensors in $V \equiv \otimes_{n} V_{n}$. Indeed, $\psi$ is determined by its values on the dense $\mathcal{G}$-orbit of $A=0$. Hence the bundle $\mathcal{W}$ is a subbundle of the trivial bundle with the fiber $V^{G}$. The $\mathrm{KZ}(\mathrm{B})$ connection reduces in this case [18] to the formula

$$
\begin{aligned}
\nabla_{\delta \mu}^{\mathrm{KZ}} \psi(0) & =\sum_{n}\left(\delta \chi_{n}\left(\partial_{\chi_{n}}-\chi_{n}^{-1} \Delta_{n}\right)+\delta z_{n}\left(\partial_{z_{n}}-\frac{1}{\kappa} H_{n}\right)\right) \psi(0) \\
\nabla \frac{\mathrm{KZ}}{\delta \mu} \psi(0) & =\sum_{n}\left({\overline{\delta \chi_{n}}}_{\bar{\chi}_{n}}+\overline{\delta z}_{n} \partial_{\bar{z}_{n}}\right) \psi(0)
\end{aligned}
$$

where

$$
\Delta_{n}=\frac{1}{2 \kappa} t_{n}^{a} t_{n}^{a}, \quad H_{n}=\sum_{m \neq n} \frac{t_{n}^{a} t_{m}^{a}}{z_{n}-z_{m}}
$$

with $t_{n}^{a}$ denoting the action of the generator $t^{a}$ in the factor $V_{n}$ of $V . \Delta_{n}$ is a number, the conformal weight assigned in the WZNW theory to the irreducible representation of $G$ in $V_{n}$ [18]. Note that, modulo the shift $k \mapsto \kappa=k+g^{\vee}, \Delta_{n}$ and $\frac{1}{\kappa} H_{n}$ may be obtained from $\frac{k}{(2 \pi i)^{2}} \delta_{n}$ and $\frac{1}{(2 \pi i)^{2}} h_{n}$, respectively, by the (geometric) quantization of the coadjoint orbits which replaces the functions $\nu_{n}^{a}$ by the operators $\frac{2 \pi}{k i} t_{n}^{a}$ so that the Poisson bracket turns into $\frac{k i}{2 \pi}$ times the commutator $\left(\frac{2 \pi}{k}\right.$ plays the role of the Planck constant). The flatness of the connection $\nabla^{\mathrm{KZ}},\left(\nabla^{\mathrm{KZ}}\right)^{2}=0$, follows from the equation $\left[H_{n}, H_{m}\right]=0$, equivalent to the classical Yang-Baxter equation (CYBE) for $\frac{t_{1}^{a} t_{2}^{a}}{z}$ :

$$
\left[\frac{t_{n}^{a} t_{m}^{a}}{z_{n}-z_{m}}, \frac{t_{m}^{a} t_{p}^{a}}{z_{m}-z_{p}}\right]+\text { cyclic permutations }=0 \text {. }
$$

\section{Genus one}

At genus one, every Riemann surface is isomorphic to an elliptic curve $E_{\tau} \equiv \mathbb{C} /(\mathbb{Z}+\tau \mathbb{Z})$ where $\tau$ is a complex number of positive imaginary part $\tau_{2}$. Denote by $\Delta\left(\Delta_{+}\right)$the set of (positive) roots of $\operatorname{Lie}(G)$, by $e_{\alpha}$ the step generators attached to the roots $\alpha$ and let $\left(\eta^{j}\right)$ be an orthonormal basis of the Cartan algebra Lie $(T)$. We set $u_{\alpha}=\operatorname{tr} u \alpha$ and $u^{j}=\operatorname{tr} u \eta^{j}$ for $u \in \operatorname{Lie}(T)$. We shall need some elliptic functions: the Jacobi theta function

$$
\vartheta_{1}(z)=-i \sum_{\ell=-\infty}^{\infty}(-1)^{\ell} \mathrm{e}^{\pi i \tau\left(\ell+\frac{1}{2}\right)^{2}+\pi i z(2 \ell+1)},
$$


the Green function $P_{x}$ of the twisted $\bar{\partial}$-operator

$$
P_{x}(z)=\frac{\vartheta_{1}^{\prime}(0) \vartheta_{1}(x+z)}{\vartheta_{1}(x) \vartheta_{1}(z)}
$$

with the properties $P_{x}(z+1)=P_{x}(z), P_{x}(z+\tau)=\mathrm{e}^{-2 \pi i x} P_{x}(z)$ and $P_{x}(z)=\frac{1}{z}+\mathcal{O}(1)$ around $z=0$, the function

$$
\rho=\vartheta_{1}^{\prime} / \vartheta_{1}
$$

s.t. $\rho(z+1)=\rho(z), \rho(z+\tau)=\rho(z)-2 \pi i, \rho(z)=\frac{1}{z}+\mathcal{O}(1)$ around $z=0$ and, finally, the Weyl-Kac denominator

$$
\Pi(u)=\mathrm{e}^{2 \pi i \tau d / 24} \prod_{\alpha \in \Delta_{+}}\left(\mathrm{e}^{\pi i u_{\alpha}}-\mathrm{e}^{-\pi i u_{\alpha}}\right) \prod_{\ell=1}^{\infty}\left[\left(1-\mathrm{e}^{2 \pi i \ell \tau}\right)^{r} \prod_{\alpha \in \Delta}\left(1-\mathrm{e}^{2 \pi i \ell \tau} \mathrm{e}^{2 \pi i u_{\alpha}}\right)\right]
$$

where $d$ denotes the dimension and $r$ the rank of $G$.

On $E_{\tau}$ a generic gauge field is in the orbit of $A_{u}=\pi u d \bar{z} / \tau_{2}$, for $u \in \operatorname{Lie}(T)$, i.e.

$$
A={ }^{h^{-1}} A_{u}^{01}=\left(h_{u} h\right)^{-1} \bar{\partial}\left(h_{u} h\right)
$$

where $h_{u}=\mathrm{e}^{\pi(u \bar{z}-\bar{u} z) / \tau_{2}}$. Consequently, the gauge fields may be parametrized by $u$ and $h$. To avoid ambiguities, we have to identify the pairs as follows

$$
(u, h) \sim\left(w u w^{-1}, w h\right) \sim\left(u+q^{\vee}, h_{q^{\vee}}^{-1} h\right) \sim\left(u+\tau q^{\vee}, h_{\tau q^{\vee}}^{-1} h\right),
$$

for $q^{\vee}$ in the coroot lattice $Q^{\vee}$ and $w$ in the the normalizer $N$ of $\operatorname{Lie}(T)$ in G. Similarly to the genus zero case, we have to solve the equation

$$
\bar{\partial}\left({ }^{h_{u} h} \Phi\right)=\sum_{n} \nu_{n} \delta_{z_{n}}
$$

where $\nu_{n}=\left(h_{u} h\right)\left(z_{n}\right) \lambda_{n}\left(h_{u} h\right)\left(z_{n}\right)^{-1}$. Decomposing $\nu_{n}=\sum_{\alpha} \nu_{n}^{-\alpha} e_{\alpha}+\nu_{n}^{0}$ with $\nu_{n}^{0}=\nu_{n}^{j} \eta^{j} \in$ $\operatorname{Lie}(T)$, we can solve the above equation if and only if $\sum_{n} \nu_{n}^{0}=0$. In that case,

$$
{ }^{h_{u} h} \Phi(z)=\left(\varphi_{0}+\sum_{n}\left(\sum_{\alpha} P_{u_{\alpha}}\left(z-z_{n}\right) \nu_{n}^{-\alpha} e_{\alpha}+\rho\left(z-z_{n}\right) \nu_{n}^{0}\right)\right) \frac{d z}{2 \pi i}
$$

for an arbitrary constant $\varphi_{0}=\varphi_{0}^{j} \eta^{j}$. Performing the symplectic reduction, we find

$$
\left.\mathcal{P}_{\mathcal{O}} \simeq\left\{\left(u, \varphi_{0}, \underline{\nu}\right) \in T^{*} \operatorname{Lie}(T) \times \underset{n}{\times} \mathcal{O}_{n}\right) \mid \sum_{n} \nu_{n}^{0}=0\right\} / N \rtimes\left(Q^{\vee}+\tau Q^{\vee}\right)
$$

where the action of $N \rtimes\left(Q^{\vee}+\tau Q^{\vee}\right)$ implements the identifications

$$
\begin{aligned}
\left(u, \varphi_{0}, \underline{\nu}\right) & \sim\left(w u w^{-1}, w \varphi_{0} w^{-1}, w \underline{\nu} w^{-1}\right) \sim\left(u+q^{\vee}, \varphi_{0},\left(h_{q^{\vee}}^{-1}\left(z_{n}\right) \nu_{n} h_{q^{\vee}}\left(z_{n}\right)\right)\right) \\
& \sim\left(u+\tau q^{\vee}, \varphi_{0},\left(h_{\tau q^{\vee}}^{-1}\left(z_{n}\right) \nu_{n} h_{\tau q^{\vee}}\left(z_{n}\right)\right)\right) .
\end{aligned}
$$


The symplectic structure of $\mathcal{P}_{\mathcal{O}}$ is that of the reduction of $\left.T^{*} \operatorname{Lie}(T) \times \underset{n}{(} \underset{\mathcal{O}_{n}}{ }\right)$ by the group $N \rtimes\left(Q^{\vee}+\tau Q^{\vee}\right)$. Now it is easy to write down the Hitchin Hamiltonians. Let us us do it for $p_{2}=\frac{1}{2}$ tr. A straightforward computation identifying the pole terms leads to

$$
h_{p_{2}}=\left\{-\sum_{n} \rho^{\prime}\left(z-z_{n}\right) \delta_{n}+\sum_{n} \rho\left(z-z_{n}\right) h_{n}+h_{0}\right\}\left(\frac{d z}{2 \pi i}\right)^{2}
$$

where, as before, $\delta_{n}=\frac{1}{2} \nu_{n}^{a} \nu_{n}^{a}$ and

$$
\begin{aligned}
& h_{0}=\frac{1}{2} \sum_{j=1}^{r} \varphi_{0}^{j} \varphi_{0}^{j}+\frac{1}{2} \sum_{m, n}\left\{\sum_{\alpha} \partial_{x} P_{u_{\alpha}}\left(z_{n}-z_{m}\right) \nu_{n}^{\alpha} \nu_{m}^{-\alpha}+\frac{1}{2} \sum_{j=1}^{r} \frac{\vartheta_{1}^{\prime \prime}}{\vartheta_{1}}\left(z_{n}-z_{m}\right) \nu_{n}^{j} \nu_{m}^{j}\right\}, \\
& h_{n}=\sum_{j=1}^{r} \nu_{n}^{j} \varphi_{0}^{j}+\sum_{m \neq n}\left(\sum_{\alpha} P_{u_{\alpha}}\left(z_{n}-z_{m}\right) \nu_{n}^{\alpha} \nu_{m}^{-\alpha}+\sum_{j=1}^{r} \rho\left(z_{n}-z_{m}\right) \nu_{n}^{j} \nu_{m}^{j}\right) .
\end{aligned}
$$

Note the similarity to the genus 0 case (3.6).

Let $\delta \mu=\delta \mu_{\bar{z}}^{z} \partial_{z} d \bar{z}$ be a Beltrami differential on $E_{\tau}$ behaving like $\mathcal{O}\left(\left(z-z_{n}\right)^{2}\right)$ around the insertions. The modified complex structure corresponds to the complex coordinate $z^{\prime}=z+\frac{z-\bar{z}}{2 i \tau_{2}} \delta \tau+\delta \xi^{z}$ s.t. $\partial_{\bar{z}} z^{\prime}=\delta \mu_{\bar{z}}^{z}$. We require that $\delta \xi^{z}(z+1)=\delta \xi^{z}(z+\tau)=\delta \xi^{z}(z)$. $\delta \tau$ is determined from the condition that the integral of $\delta \mu_{\bar{z}}^{z}$ over $E_{\tau}$ is equal to that of $\frac{i}{2 \tau_{2}} \delta \tau . \delta \xi^{z}$ is unique up to an additive constant. Note that $z^{\prime}(z+1)=z^{\prime}(z)+1$ whereas $z^{\prime}(z+\tau)=z^{\prime}+\tau^{\prime}$ where $\tau^{\prime}=\tau+\delta \tau$. Hence the deformed curve is isomorphic to $E_{\tau^{\prime}}$ with the punctures moved to $z_{n}^{\prime}=z_{n}+\delta z_{n}$ and the first jets of local parameters changed to $\chi_{n}^{\prime}=\chi_{n}+\delta \chi_{n}$ with

$$
\delta z_{n}=\frac{z_{n}-\bar{z}_{n}}{2 i \tau_{2}} \delta \tau+\delta \xi^{z}\left(z_{n}\right), \quad \chi_{n}^{-1} \delta \chi_{n}=\frac{\delta \tau}{2 i \tau_{2}}+\partial_{z} \delta \xi^{z}\left(z_{n}\right) .
$$

Again by a straightforward calculation substituting $\delta \mu_{\bar{z}}^{z}=\partial_{\bar{z}} z^{\prime}$, cutting out small balls around points $z_{n}$ and integrating by parts, we obtain

$$
h_{\delta \mu}=\int_{E_{\tau}} h_{p_{2}} \delta \mu=\frac{1}{2 \pi i} \sum_{n}\left(\delta_{n} \chi_{n}^{-1} \delta \chi_{n}+h_{n} \delta z_{n}+\frac{1}{2 \pi i} h_{0} \delta \tau\right) .
$$

The quantum states $\psi$ at genus one may be characterized by giving holomorphic functions $\widetilde{\psi}(u)$ on $\operatorname{Lie}(T)$ with values in $V^{T}$, the subspace of $T$-invariant tensors in the product $V$ of the representation spaces,

$$
\widetilde{\psi}(u)=\Pi(u) \mathrm{e}^{-\pi k \operatorname{tr} u^{2} /\left(2 \tau_{2}\right)} \underset{n}{\otimes}\left(\mathrm{e}^{-\pi\left(z_{n}-\bar{z}_{n}\right) u / \tau_{2}}\right)_{n} \psi\left(A_{u}\right) .
$$

The KZB connection takes the form [6, 7, 10, 9, 8]

$$
\begin{aligned}
& \nabla_{\delta \mu}^{\mathrm{KZB}} \widetilde{\psi}=\sum_{n}\left(\delta \chi_{n}\left(\partial_{\chi_{n}}-\chi_{n}^{-1} \Delta_{n}\right)+\delta z_{n}\left(\partial_{z_{n}}-\frac{1}{\kappa} H_{n}\right)+\delta \tau\left(\partial_{\tau}-\frac{1}{2 \pi i \kappa} H_{0}\right)\right) \widetilde{\psi} \\
& \nabla_{\delta \mu}^{\mathrm{KZB}} \widetilde{\psi}=\sum_{n}\left({\overline{\delta \chi_{n}}}_{\bar{\chi}_{n}}+\overline{\delta z}_{n} \partial_{\bar{z}_{n}}+\overline{\delta \tau} \partial_{\bar{\tau}}\right) \widetilde{\psi}
\end{aligned}
$$


where $\Delta_{n}$ is as before and the operators $\frac{1}{k} H_{0}$ and $\frac{1}{k} H_{n}$ are obtained from the Hamiltonians $\frac{k}{(2 \pi i)^{2}} h_{0}$ and $\frac{k}{(2 \pi i)^{2}} h_{n}$ by the replacement

$$
\varphi_{0}^{j} \mapsto \frac{2 \pi}{k i} \partial_{u^{j}}, \quad \mu_{n}^{\alpha} \mapsto \frac{2 \pi}{k i} e_{\alpha n}, \quad \mu_{n}^{j} \mapsto \frac{2 \pi}{k i} \eta_{n}^{j},
$$

i.e. by the geometric quantization. The resulting Hamiltonians $H_{0}$ and $H_{n}$ act on general meromorphic functions on $\operatorname{Lie}(T)$ with values in $V^{T}$. The flatness of the KZB connection is ensured by their commutation: $\left[H_{n}, H_{m}\right]=0$, for $n, m=0,1, \ldots$, following from the so called dynamical CYBE [10].

\section{Genus two}

\subsection{Curve and its Jacobian}

Let $\Sigma$ be a curve of genus 2. Choosing a marking, i.e. a symplectic homology basis $\left(A^{a}, B^{a}\right), a=1,2$, on $\Sigma$, we may fix the corresponding basis $\left(\omega^{a}\right)$ of the holomorphic 1,0 -forms (abelian differentials) s.t. $\int_{A^{a}} \omega^{b}=\delta^{a b}$. The $B^{a}$-periods of the abelian differentials give rise to the symmetric period matrix $\tau, \tau^{a b}=\int_{B^{a}} \omega^{b}$, with a positive imaginary part. The map

$$
\Sigma \ni x \mapsto z(x)=\frac{\omega^{2}(x)}{\omega^{1}(x)}
$$

realizes $\Sigma$ as a double covering of $\mathbb{P}^{1}$ ramified over six Weierstrass points $x_{n}$ or as a hyperelliptic curve given by the equation

$$
y^{2}=\prod_{n=1}^{6}\left(z-z_{n}\right) .
$$

The coordinates $z_{n}=z\left(x_{n}\right)$ are assumed to be finite. This may be always achieved by an appropriate choice of the marking. Curve $\Sigma$ may be viewed as composed of the points $(y, z)$ and of two points at infinity. The covering of $\mathbb{P}^{1}$ is $(y, z) \mapsto z$. In this representation, the holomorphic 1,0-forms and the holomorphic quadratic differentials on $\Sigma$ have the form

$$
\omega=(a+b z) d z / y, \quad \beta=\left(a+b z+c z^{2}\right)(d z)^{2} / y^{2},
$$

respectively. In particular,

$$
\omega^{1} \propto d z / y, \quad \omega^{2} \propto z d z / y
$$

with the same proportionality constant.

The Jacobian $J^{1}$ of the degree 1 holomorphic line bundles may be represented as $\mathbb{C}^{2} /\left(\mathbb{Z}^{2}+\tau \mathbb{Z}^{2}\right)$. In particular, the spin structures $L, L^{2}=K$, correspond to points $e+\tau e^{\prime}$ with $e \in \frac{1}{2} \mathbb{Z}^{2} / \mathbb{Z}^{2}$. There are 6 odd spin structures $e_{n}+\tau e_{n}^{\prime}$ labeled by the Weierstrass

points $z_{n}$, the zeros of the holomorphic sections they admit, and 10 even spin structures without holomorphic sections. 


\subsection{Theta functions}

The degree 1 bundles with holomorphic sections form the theta-divisor in $J^{1}$. The holomorphic sections of the $k^{\text {th }}$-power of the corresponding theta bundle over $J^{1}$ may be represented by the holomorphic theta functions of degree $k$ on $\mathbb{C}^{2}$ defined by the relations

$$
\mathrm{e}^{\pi i k n \cdot \tau n+2 \pi i k n \cdot u} \theta(u+m+\tau n)=\theta(u)
$$

for $m, n \in \mathbb{Z}^{2}$. They form the space $\Theta_{k}$ of dimension $k^{2}$. For $k=1$ there is a single (up to normalization) theta function

$$
\vartheta(u)=\sum_{n \in \mathbb{Z}^{2}} \mathrm{e}^{\pi i n \cdot \tau n+2 \pi i n \cdot u},
$$

the Riemann theta function. For $k=2$ there are four independent $\theta$-functions. One can take them as

$$
\theta_{e}(u)=\sum_{n \in \mathbb{Z}^{2}} \mathrm{e}^{2 \pi i(n+e) \cdot \tau(n+e)+4 \pi i(n+e) \cdot u}
$$

for $e \in \frac{1}{2} \mathbb{Z}^{2} / \mathbb{Z}^{2}$.

$$
\vartheta(u+v) \vartheta(u-v)=\sum_{e} \theta_{e}(u) \theta_{e}(v)
$$

is a second order theta function in both $u$ and $v$. The map $v \mapsto \vartheta(\cdot+v) \vartheta(\cdot-v)$ determines an embedding of the Kummer surface $J^{1} / \mathbb{Z}_{2} \cong \mathbb{C}^{2} /\left(\mathbb{Z}^{2}+\tau \mathbb{Z}^{2}\right) / \mathbb{Z}_{2}$ onto a quartic surface $\mathcal{K}$ in the 3 -dimensional projective space $\mathbb{P}_{2}\left(\mathbb{Z}_{2}\right.$ maps the degree 1 line bundles $L$ into $L^{-1} K$ or $v \in \mathbb{C}^{2}$ into $\left.-v\right)$.

The double theta function (5.8) determines a non-degenerate symmetric quadratic form on the space $\Theta_{2}^{*}$ dual to $\Theta_{2}$. It permits to identify $\Theta_{2}^{*}$ with $\Theta_{2}$ by sending $\phi \in \Theta_{2}^{*}$ to $\iota(\phi) \in \Theta_{2}$ defined by

$$
\iota(\phi)(u)=\langle\vartheta(u+\cdot) \vartheta(u-\cdot), \phi\rangle .
$$

The identification exchanges the basis $\left(\theta_{e}\right)$ of $\Theta_{2}$ with the dual basis $\left(\theta_{e}^{*}\right)$ and the Kummer quartic $\mathcal{K}$ with its dual version $\mathcal{K}^{*} \subset \mathbb{P} \Theta_{2}^{*}$. $\mathcal{K}^{*}$ is composed of linear forms proportional to the evaluation forms $\phi_{u}$ defined by

$$
\left\langle\theta, \phi_{u}\right\rangle=\theta(u) .
$$

The group $\left(\frac{1}{2} \mathbb{Z} / \mathbb{Z}\right)^{4}$ of spin structures acts on $\Theta_{k}$ for even $k$ by endomorphisms $U_{e, e^{\prime}}$ defined by

$$
\left(U_{e, e^{\prime}} \theta\right)(u)=\mathrm{e}^{\pi i k e^{\prime} \cdot \tau e^{\prime}+2 \pi i k e^{\prime} \cdot u} \theta\left(u+e+\tau e^{\prime}\right) .
$$

For $k$ not divisible by 4 this action is only projective: $U_{e, e^{\prime}} U_{f, f^{\prime}}=(-1)^{4 e \cdot f^{\prime}} U_{e+f, e^{\prime}+f^{\prime}}$ and it lifts to a Heisenberg group. For $k=2$,

$$
U_{e, e^{\prime}} \theta_{e^{\prime \prime}}=(-1)^{4 e \cdot e^{\prime \prime}} \theta_{e^{\prime}+e^{\prime \prime}} .
$$

The action of $U_{e, e^{\prime}}$ preserves the Kummer quartic $\mathcal{K} \subset \mathbb{P} \Theta_{2}$ and the action of the transposed endomorphisms $U_{e, e^{\prime}}^{t}$ preserves $\mathcal{K}^{*}$. 


\subsection{Moduli space of $S L_{2}$-bundles}

In the fundamental paper [21], Narasimhan and Ramanan proved that the moduli space $\mathcal{N}_{s}$ of the stable $S L_{2}$ holomorphic bundles is canonically isomorphic to $\mathbb{P} \Theta_{2} \backslash \mathcal{K}$. The isomorphism associates to an $S L_{2}$-bundle $E$ the second order theta function $\theta$ vanishing at the points $u \in \mathbb{C}^{2}$ corresponding to the duals of the line-subbundles of the rank 2 bundle associated to $E$. In other words, if $A$ is the gauge field whose $\mathcal{G}$-orbit corresponds to $E$ and if $L_{u}$ denotes the degree 1 line bundle corresponding to $u \in \mathbb{C}^{2} /\left(\mathbb{Z}^{2}+\tau \mathbb{Z}^{2}\right)$ then the theta function $\theta$ associated to $E$ vanishes at $u$ if and only if there exists a pair $s=\left(s_{1}, s_{2}\right)$ composed of sections of $L_{u}$ s.t. $(\bar{\partial}+A) s=0$. The semistable compactification of the moduli space $\mathcal{N}_{s}$ is

$$
\mathcal{N}_{s s} \cong \mathbb{P} \Theta_{2}
$$

and, exceptionally, it is smooth. The points of the Kummer quartic $\mathcal{K}$ represent (classes of) the semistable but not stable bundles. Hence for $G=S L_{2}$ the phase space of the Hitchin system on the genus 2 curve with no insertions is

$$
T^{*} \mathcal{N}_{s s} \cong T^{*} \mathbb{P} \Theta_{2} \cong\left\{(\theta, \phi) \in \Theta_{2} \times \Theta_{2}^{*} \mid \theta \neq 0,\langle\theta, \phi\rangle=0\right\} / \mathbb{C}^{\times}
$$

with the action of $t \in \mathbb{C}^{\times}$given by $(\theta, \phi) \mapsto\left(t \theta, t^{-1} \phi\right)$. As a symplectic space, it is the symplectic reduction of $T^{*}\left(\Theta_{2} \backslash\{0\}\right)$ by the action of $\mathbb{C}^{\times}$. Using the bases $\left(\theta_{e}\right)$ and $\left(\theta_{e}^{*}\right)$ to decompose

$$
\theta=\sum q_{e} \theta_{e}, \quad \phi=\sum p_{e} \theta_{e}^{*},
$$

we may represent $T^{*} \mathcal{N}_{s s}$ as the space of pairs $(q, p) \in \mathbb{C}^{4} \times \mathbb{C}^{4}, q \neq 0, q \cdot p=0$, with the identification $(q, p) \sim\left(t q, t^{-1} p\right)$ and the symplectic form induced from $d p \cdot d q$.

\subsection{Hitchin map for $G=S L_{2}$}

The Hitchin map $h_{p_{2}}: T^{*} \mathbb{P} \Theta_{2} \rightarrow H^{0}\left(K^{2}\right)$ appears to take a particularly simple form resembling the genus 0 formula (3.6):

$$
h_{p_{2}}=\frac{1}{2} \sum_{\substack{n, m=1 \\ n \neq m}}^{6} \frac{r_{n m}}{\left(z-z_{n}\right)\left(z-z_{m}\right)}\left(\frac{d z}{2 \pi i}\right)^{2}=\sum_{n=1}^{6} \frac{h_{n}}{z-z_{n}}\left(\frac{d z}{2 \pi i}\right)^{2}
$$

where

$$
r_{n m}(\theta, \phi)=\frac{1}{16}\left\langle U_{e_{n}, e_{n}^{\prime}} \theta, U_{e_{m}, e_{m}^{\prime}}^{t} \phi\right\rangle\left\langle U_{e_{m}, e_{m}^{\prime}} \theta, U_{e_{n}, e_{n}^{\prime}}^{t} \phi\right\rangle
$$

(the last two factors on the right hand side coincide modulo sign) and

$$
h_{n}=\sum_{m \neq n}^{6} \frac{r_{n m}}{z_{n}-z_{m}} .
$$

With the help of Eq. (5.12), $r_{n m}$ 's may be rewritten in the language of $q$ 's and $p$ 's as explicit homogeneous polynomials of order 2 in $q_{e}$ and in $p_{e}$, see below. The identity

$$
\sum_{m \neq n} r_{n m}=0
$$


holding for each $n$ guarantees that $\prod_{n=1}^{6}\left(z-z_{n}\right) \sum_{\substack{n, m=1 \\ n \neq m}}^{6} \frac{r_{n m}}{\left(z-z_{n}\right)\left(z-z_{m}\right)}$ is a quadratic polynomial in $z$. It follows that the right hand side of Eq. (5.16) determines a quadratic differential on $\Sigma$ of the general form given by Eq. (5.3).

The equality (5.16) is not immediate. It was established in four steps. We shall only enumerate them here. The two first crucial steps were performed in [14. It was shown there that for any $\theta \neq 0$ and $u \in \mathbb{C}^{2}$ s.t. $\theta(u)=0$,

$$
h_{p_{2}}\left(\theta, \phi_{u}\right)=-\frac{1}{16 \pi^{2}}\left(\partial_{u^{a}} \theta(u) \omega^{a}\right)^{2} .
$$

The above equation describes the quadratic polynomial $h_{p_{2}}(\theta, \cdot)$ (with values in $H^{0}\left(K^{2}\right)$ ) on the quartic $\mathcal{K}_{\theta}^{*}=\mathcal{K}^{*} \cap \mathbb{P} \theta^{\perp}$ in the projectivized subspace of $\Theta_{2}^{*}$ perpendicular to $\theta$. In principal, it determines $h_{p_{2}}$ completely. It was observed then that the above formula implies that for each Weierstrass point $z_{n}$ the conic

$$
\mathcal{C}_{n}=\left\{\mathbb{C}^{\times} \phi \in \mathbb{P} \theta^{\perp}\left|h_{p_{2}}(\theta, \phi)\right|_{z_{n}}=0\right\}
$$

is in fact the union of two bitangents to $\mathcal{K}_{\theta}^{*}$. The explicit equations for the bitangents to the Kummer quartics known since about a century permitted then to establish Eq. (5.16) up to multiplication by a $\theta$-dependent factor [14. The other steps in the proof of the formula (5.16) were taken in [13] were it was established that the Hitchin map $h_{p_{2}}$ possesses the important self-duality property:

$$
h_{p_{2}}\left(\iota(\phi), \iota^{-1}(\theta)\right)=h_{p_{2}}(\theta, \phi)
$$

or that $h_{p_{2}}(q, p)=h_{p_{2}}(p, q)$ in the language of (5.15). This property, far from obvious in the original formulation of the Hitchin system, restricted the ambiguity on the right hand side of Eq. (5.16) to a (possibly curve-dependent) constant factor. The latter was fixed in [13] by a tedious calculation of $h_{p_{2}}$ at special points of $\mathcal{K} \times \mathcal{K}^{*}$.

\subsection{Deformations of complex structure}

For the hyperelliptic curve, the variations of the complex structure described by the Beltrami differentials $\delta \mu$ on $\Sigma$ change the images $z_{n}$ of the ramification points of the covering of $\mathbb{P}^{1}$. Let us find these changes. Let

$$
\omega^{\prime a}=\omega^{a}+\delta \omega^{a}+\bar{\delta} \omega^{a}
$$

denote a deformed basis $\left(\omega^{\prime a}\right)$ of the abelian differentials with $\delta \omega^{a}$ of 1,0 -type and $\bar{\delta} \omega^{a}$ of 0,1-type in the original complex structure. $\delta \omega^{a}$ and $\bar{\delta} \omega^{a}$ have to satisfy the relations

$$
\bar{\delta} \omega^{a}=\omega^{a} \delta \mu, \quad \bar{\partial}\left(\delta \omega^{a}\right)=-\partial\left(\omega^{a} \delta \mu\right)
$$

stating, respectively, that $\omega^{\prime a}$ is of the 1,0-type in the deformed structure and that it is a closed form. The equation for $\delta \omega^{a}$ always has solutions. They are defined modulo abelian differentials and the ambiguity may be fixed by demanding that $\int_{A^{a}} \omega^{\prime b}=\delta^{a b}$. The deformed covering map onto $\mathbb{P}^{1}$ is then

$$
z^{\prime}(x)=\frac{\omega^{\prime 2}(x)}{\omega^{\prime 1}(x)}=z(x)+\frac{\delta \omega^{2}}{\omega^{1}}(x)-z(x) \frac{\delta \omega^{1}}{\omega^{1}}(x) .
$$


The ramification points $x_{n}^{\prime}$ of the map $z^{\prime}$ are determined by solving the equation

$$
\partial^{\prime} z^{\prime}\left(x_{n}^{\prime}\right)=0
$$

which, upon rewriting $x_{n}^{\prime}=x_{n}+\delta x_{n}$ becomes

$$
(\partial)^{2} z\left(x_{n}\right) \delta x_{n}+\partial\left(\frac{\delta \omega^{2}}{\omega^{1}}\right)\left(x_{n}\right)-\partial\left(z \frac{\delta \omega^{1}}{\omega^{1}}\right)\left(x_{n}\right)=0 .
$$

Since $\bar{\partial} z\left(x_{n}\right)=0$, the quadratic differential $(\partial)^{2} z\left(x_{n}\right)$ is well defined. Besides, since the ramification points are isolated, it does not vanish so that one may solve the above equations for $\delta x_{n}$. The ramification points $x_{n}^{\prime}$ are mapped to

$$
z_{n}^{\prime}=z^{\prime}\left(x_{n}+\delta x_{n}\right)=z_{n}+\frac{\delta \omega^{2}}{\omega^{1}}\left(x_{n}\right)-z_{n} \frac{\delta \omega^{1}}{\omega^{1}}\left(x_{n}\right) .
$$

We infer that

$$
\delta z_{n} \equiv z_{n}^{\prime}-z_{n}=\frac{\delta \omega^{2}}{\omega^{1}}\left(x_{n}\right)-z_{n} \frac{\delta \omega^{1}}{\omega^{1}}\left(x_{n}\right)
$$

are the variations of $z_{n}$ corresponding to the Beltrami differential $\delta \mu$.

We may now find the values of the Hitchin Hamiltonians $h_{\delta \mu}=\int_{\Sigma} h_{p_{2}} \delta \mu$ related to the Beltrami differentials. Note that, by virtue of Eqs. (5.25) and (5.24),

$$
\begin{gathered}
\bar{\partial}(\delta z) \equiv \bar{\partial}\left(z^{\prime}-z\right)=\bar{\partial}\left(\delta \omega^{2}\right) / \omega^{1}-z \bar{\partial}\left(\delta \omega^{1}\right) / \omega^{1}=-\partial\left(\omega^{2} \delta \mu\right) / \omega^{1} \\
+z \partial\left(\omega^{1} \delta \mu\right) / \omega^{1}=-\partial\left(z \omega^{1} \delta \mu\right) / \omega^{1}+z \partial\left(\omega^{1} \delta \mu\right) / \omega^{1}=(d z) \delta \mu .
\end{gathered}
$$

A straightforward integration by parts over the region in $\Sigma$ without small balls around the Weierstrass points $x_{n}$ and around 2 points at infinity gives now:

$$
\begin{array}{r}
h_{\delta \mu}=\int_{\Sigma} \sum_{n=1}^{6} \frac{h_{n}}{z-z_{n}}\left(\frac{d z}{2 \pi i}\right)^{2} \delta \mu=\left(\frac{1}{2 \pi i}\right)^{2} \int_{\Sigma} \sum_{n=1}^{6} \frac{h_{n}}{z-z_{n}} d z \bar{\partial}(\delta z) \\
=\frac{1}{\pi i} \sum_{n=1}^{6} h_{n} \delta z_{n} .
\end{array}
$$

The comparison with Eq. (3.8) shows an additional factor 2 which comes from the double covering.

\subsection{Relation to Neumann systems}

The Poisson-commutation of the Hamiltonians $h_{n}$ (of which any 3 give independent action variables of our integrable system) is equivalent to the CYB-like equations

$$
\left\{\frac{r_{n m}}{z_{n}-z_{m}}, \frac{r_{m p}}{z_{m}-z_{p}}\right\}+\text { cycl. }=0, \quad\left\{\frac{r_{n m}}{z_{n}-z_{m}}, \frac{r_{p q}}{z_{p}-z_{q}}\right\}=0
$$

for $\{n, m\} \cap\{p, q\}=\emptyset$. The above relations may may be directly checked, as noticed in [14]. The more recent observations of the paper [15] on the Knizhnik-ZamolodchikovBernard connection in the same setup (see below) permit to identify the integrable system 
with the Hamiltonians $h_{n}$ of Eq. (5.18): it is a modified version of the classical genus 2 Neumann systems [23, 19, 1] whose original version is also rooted in the modular geometry of hyperelliptic curves. This goes as follows.

The phase space $T^{*} \mathbb{P}^{3}$ (without the zero section) may be identified with the coadjoint orbit $\mathcal{O}_{1}$ of the complex group $S L_{4}$ composed of the traceless rank 1 matrices $|q\rangle\langle p|$. The action of $S L_{4}$ in $\wedge^{2} \mathbb{C}^{4}$ preserves the quadratic form induced by the exterior product on $\wedge^{2} \mathbb{C}^{4}$ and the identification $\wedge^{4} \mathbb{C}^{4} \cong \mathbb{C}^{1}$. It leads to the double covering $S L_{4} \rightarrow S O_{6}$ if we choose in $\wedge^{2} \mathbb{C}^{4}$ the Plücker basis turning the quadratic form into the sum of squares. The inverse relation is the complexified version of the twistor calculus. Upon the identification of $s l_{4}$ with ${s o_{6}}_{6}$, the coadjoint orbit $\mathcal{O}_{1}$ becomes the one composed of (complex) rank 2 antisymmetric matrices $J=\left(J_{n m}\right)$ of square zero: $J^{2}=0$. Such matrices are of the form $J_{n m}=Q_{n} P_{m}-P_{n} Q_{m}$ with vectors $P, Q \in \mathbb{C}^{6}$ spanning an isotropic subspace, i.e. with $Q^{2}=Q \cdot P=P^{2}=0$. Given $(q, p)$ with $q \cdot p=0$, in order to find the corresponding pair $(Q, P)$, it is enough to complete vector $q$ to a basis $\left(q, f_{1}, f_{2}, f_{3}\right)$ of $\mathbb{C}^{4}$ s.t. $f_{1} \cdot p=f_{2} \cdot p=0$ and $f_{3} \cdot p=1$ and to set

$$
Q=q \wedge f_{1}, \quad R=f_{2} \wedge f_{3}, \quad P=q \wedge f_{2} / Q \cdot R
$$

in the language of $\wedge^{2} \mathbb{C}^{4} \cong \mathbb{C}^{6}$. In the Plücker coordinates, we may take

$$
\begin{aligned}
& Q_{1}=-\left(q_{1} p_{3}+q_{4} p_{2}\right), \quad Q_{2}=i\left(q_{1} p_{3}-q_{4} p_{2}\right), \quad Q_{3}=-i\left(q_{1} p_{2}+q_{4} p_{3}\right), \\
& Q_{4}=q_{1} p_{2}-q_{4} p_{3}, \quad Q_{5}=q_{2} p_{2}+q_{3} p_{3}, \quad Q_{6}=i\left(q_{2} p_{2}+q_{3} p_{3}\right), \\
& P_{1}=-\frac{1}{2} \frac{q_{2} p_{4}+q_{3} p_{1}}{q_{2} p_{2}+q_{3} p_{3}}, \quad P_{2}=\frac{i}{2} \frac{q_{2} p_{4}-q_{3} p_{1}}{q_{2} p_{2}+q_{3} p_{3}}, \quad P_{3}=\frac{i}{2} \frac{q_{3} p_{4}+q_{2} p_{1}}{q_{2} p_{2}+q_{3} p_{3}}, \\
& P_{4}=-\frac{1}{2} \frac{q_{3} p_{4}-q_{2} p_{1}}{q_{2} p_{2}+q_{3} p_{3}}, \quad P_{5}=\frac{1}{2}, \quad P_{6}=-\frac{i}{2}
\end{aligned}
$$

where $q_{1} \equiv q_{(0,0)}, q_{2} \equiv q_{\left(\frac{1}{2}, 0\right)}, q_{3} \equiv q_{\left(0, \frac{1}{2}\right)}, q_{4} \equiv q_{\left(\frac{1}{2}, \frac{1}{2}\right)}$ and similarly for $p$ 's. In terms of $(Q, P)$, the symplectic form is $d P \cdot d Q$. The functions $J_{n m}$ on $\mathcal{O}_{1}$ have the Poisson bracket

$$
\begin{array}{ll}
\left\{J_{n m}, J_{m p}\right\}=-J_{n p} & \text { for } n, m, p \quad \text { different } \\
\left\{J_{n m}, J_{p q}\right\}=0 & \text { for } n, m, p, q \text { different }
\end{array}
$$

A straightforward check shows now that

$$
\begin{aligned}
J_{12}=\frac{i}{2}\left(q_{1} p_{1}+q_{2} p_{2}-q_{3} p_{3}-q_{4} p_{4}\right), & J_{13}=-\frac{i}{2}\left(q_{1} p_{4}-q_{2} p_{3}-q_{3} p_{2}-q_{4} p_{1}\right), \\
J_{14}=\frac{1}{2}\left(q_{1} p_{4}+q_{2} p_{3}-q_{3} p_{2}-q_{4} p_{1}\right), & J_{15}=-\frac{1}{2}\left(q_{1} p_{3}-q_{2} p_{4}-q_{3} p_{1}+q_{4} p_{2}\right), \\
J_{16}=\frac{i}{2}\left(q_{1} p_{3}+q_{2} p_{4}+q_{3} p_{1}+q_{4} p_{2}\right), & J_{23}=-\frac{1}{2}\left(q_{1} p_{4}-q_{2} p_{3}+q_{3} p_{2}-q_{4} p_{1}\right), \\
J_{24}=-\frac{i}{2}\left(q_{1} p_{4}+q_{2} p_{3}+q_{3} p_{2}+q_{4} p_{1}\right), & J_{25}=\frac{i}{2}\left(q_{1} p_{3}-q_{2} p_{4}+q_{3} p_{1}-q_{4} p_{2}\right), \\
J_{26}=\frac{1}{2}\left(q_{1} p_{3}+q_{2} p_{4}-q_{3} p_{1}-q_{4} p_{2}\right), & J_{34}=-\frac{i}{2}\left(q_{1} p_{1}-q_{2} p_{2}+q_{3} p_{3}-q_{4} p_{4}\right), \\
J_{35}=-\frac{i}{2}\left(q_{1} p_{2}+q_{2} p_{1}+q_{3} p_{4}+q_{4} p_{3}\right), & J_{36}=-\frac{1}{2}\left(q_{1} p_{2}-q_{2} p_{1}-q_{3} p_{4}+q_{4} p_{3}\right), \\
J_{45}=\frac{1}{2}\left(q_{1} p_{2}-q_{2} p_{1}+q_{3} p_{4}-q_{4} p_{3}\right), & J_{46}=-\frac{i}{2}\left(q_{1} p_{2}+q_{2} p_{1}-q_{3} p_{4}-q_{4} p_{3}\right), \\
J_{56}=\frac{i}{2}\left(q_{1} p_{1}-q_{2} p_{2}-q_{3} p_{3}+q_{4} p_{4}\right) &
\end{aligned}
$$


and that

$$
r_{n m}=-\frac{1}{4}\left(J_{n m}\right)^{2} .
$$

The equations (5.32) assuring that the Hamiltonians $h_{n}=-\frac{1}{4} \sum_{m \neq n} \frac{J_{n m}^{2}}{z_{n}-z_{m}}$ Poisson-commute follow directly from the $\mathrm{so}_{6}$ algebra (5.34), (5.35). The original Neumann systems are very similar but involve the coadjoint orbits of $S O_{N}$ composed from rank 2 antisymmetric matrices of square $\neq 0$ [1]. Such orbits, contrary to the one we consider, have nontrivial standard real forms.

\subsection{Lax matrix approach}

Although the change of the orbit modifies dimensional counts and many details, the methods used in the analysis of the Neumann systems, in particular the Lax method developed in [1], generalize with minor variations to our system and permit to find explicitly the angle variables of the genus 2 Hitchin system. The Lax matrix may be taken as $L(\zeta)=\left(L_{n m}(\zeta)\right)$ with

$$
L_{n m}(\zeta)=\zeta J_{n m}+z_{n} \delta_{n m}
$$

As in [1], the Poisson brackets (5.34), (5.35) may be rewritten in the matrix form as

$$
\left\{L(\zeta) \otimes 1,1 \otimes L\left(\zeta^{\prime}\right)\right\}=\left[L(\zeta) \otimes 1, r^{-}\left(\zeta, \zeta^{\prime}\right)\right]-\left[1 \otimes L\left(\zeta^{\prime}\right), r^{+}\left(\zeta, \zeta^{\prime}\right)\right]
$$

where the $r$-matrices

$$
r^{ \pm}\left(\zeta, \zeta^{\prime}\right)=\frac{\zeta \zeta^{\prime}}{\zeta+\zeta^{\prime}} C \pm \frac{\zeta \zeta^{\prime}}{\zeta-\zeta^{\prime}} T
$$

with $C_{m n, q p}=\delta_{m q} \delta_{n p}$ and $T_{m n, q p}=\delta_{m p} \delta_{n q}$ satisfy the CYBE. The above form of the Poisson bracket implies immediately that

$$
\left\{\operatorname{tr} L(\zeta)^{\ell}, \operatorname{tr} L\left(\zeta^{\prime}\right)^{\ell^{\prime}}\right\}=0
$$

for all $\zeta, \zeta^{\prime}$. Since

$$
\frac{d^{2}}{d^{2} \zeta} \operatorname{tr} L(0)^{\ell}=2 \ell \sum_{\substack{n, m=1 \\ n \neq m}}^{6} z_{n}^{\ell-1} \frac{J_{n m}^{2}}{z_{n}-z_{m}}
$$

the Hamiltonians $h_{n}=-\frac{1}{4} \sum_{m \neq n} \frac{J_{n m}^{2}}{z_{n}-z_{m}}$ may be expressed as combinations of the quantities $\operatorname{tr} L(\zeta)^{\ell}$. It is not difficult to see that the converse is also true.

More generally, Eq. (5.40) implies that

$$
\left\{\operatorname{tr} L(\zeta)^{\ell}, L\left(\zeta^{\prime}\right)\right\}=\left[M_{\ell}\left(\zeta, \zeta^{\prime}\right), L\left(\zeta^{\prime}\right)\right]
$$

with

$$
M_{\ell}\left(\zeta, \zeta^{\prime}\right)=\ell \frac{\zeta \zeta^{\prime}}{\zeta-\zeta^{\prime}} L(\zeta)^{\ell-1}+\ell \frac{\zeta \zeta^{\prime}}{\zeta+\zeta^{\prime}} L(-\zeta)^{\ell-1}
$$


It follows that the commuting time evolutions of the Lax matrix $L(\zeta)$ generated by the Hamiltonians $h_{n}$,

$$
\delta_{n} L(\zeta)=\left\{h_{n}, L(\zeta)\right\} \delta t_{n}
$$

are isospectral. In other words, the spectral curve $\mathcal{S}$ given by the characteristic equation

$$
\operatorname{det}(L(\zeta)-z)=0
$$

is left invariant by the dynamics generated by any of the Hamiltonians $h_{n}$. An easy calculation using the fact that the matrix $J$ has rank 2 gives

$$
\operatorname{det}(L(\zeta)-z)=\prod_{n=1}^{6}\left(z-z_{n}\right)\left(1+\frac{1}{2} \zeta^{2} \sum_{\substack{n, m=1 \\ n \neq m}}^{6} \frac{J_{n m}^{2}}{\left(z-z_{n}\right)\left(z-z_{m}\right)}\right) .
$$

Upon the substitution $\sigma=\frac{1}{i \zeta} \prod_{n}\left(z-z_{n}\right)$, the characteristic equation (5.46) becomes

$$
\sigma^{2}=\frac{1}{2} \prod_{n=1}^{6}\left(z-z_{n}\right)^{2} \sum_{\substack{n, m=1 \\ n \neq m}}^{6} \frac{J_{n m}^{2}}{\left(z-z_{n}\right)\left(z-z_{m}\right)} \equiv P(z) .
$$

Since $P(z)$ is a polynomial in $z$ of order 8 (see the remark after Eq. (5.19)), this is the equation of a hyperelliptic curve $\mathcal{S}$ of genus 3 composed of pairs $(\sigma, z)$ and 2 points $p_{\infty}^{ \pm}$ corresponding to $z=\infty$. We shall consider only such points of the phase space that $\mathcal{S}$ is smooth. The 1,0-forms

$$
\Omega^{b}=z^{b} d z / \sigma
$$

with $b=0,1,2$ form a basis of the abelian differentials on $\mathcal{S}$.

We shall search for the eigenvectors $X=\left(X_{n}\right)$ of the Lax matrix. This will allow to adapt the arguments described in great detail in Sect. 4 of 19 to the present case. The eigenvector equations

$$
\zeta J_{n m} X_{m}=\zeta Q_{n}(P \cdot X)-\zeta P_{n}(Q \cdot X)=\left(z-z_{n}\right) X_{n}
$$

imply that

$$
\left(z-z_{n}\right) X_{n}=a Q_{n}+b P_{n}
$$

Upon multiplication by $\sigma=\frac{1}{2} \zeta^{-1} \prod_{n}\left(z-z_{n}\right)$, Eq. (5.50) becomes a system of 2 linear equations for $a$ and $b$ :

$$
\begin{aligned}
& (\sigma+V) a+i W b=0 \\
& -i U a+(\sigma-V) b=0
\end{aligned}
$$

where

$$
U(z)=\prod_{n=1}^{6}\left(z-z_{n}\right) \sum_{n=1}^{6} \frac{Q_{n}^{2}}{z-z_{n}}
$$




$$
\begin{aligned}
& V(z)=i \prod_{n=1}^{6}\left(z-z_{n}\right) \sum_{n=1}^{6} \frac{Q_{n} P_{n}}{z-z_{n}} \\
& W(z)=\prod_{n=1}^{6}\left(z-z_{n}\right) \sum_{n=1}^{6} \frac{P_{n}^{2}}{z-z_{n}}
\end{aligned}
$$

are $4^{\text {th }}$-order polynomials in $z$. The non-trivial solution exists if

$$
\sigma^{2}=U(z) W(z)+V(z)^{2}=P(z)
$$

where the last equality follows by a straightforward check. The system (5.52) of linear equations defines a holomorphic line subbundle $L$ of the rank 2 bundle $W=\mathbb{C}^{2} \otimes \mathcal{O}\left(4 p_{\infty}^{+}+\right.$ $4 p_{\infty}^{-}$) over the hyperelliptic curve $\mathcal{S}$ (the coefficients behave as $z^{4}$ at infinity). As solutions of (5.52) we may take for example

$$
a=\sigma-V(z), \quad b=i U(z) \quad \text { or } \quad a=-i W(z), \quad b=\sigma+V(z) .
$$

Since $a$ and $b$ are proportional to $z^{4}$ at infinity, they define holomorphic sections of $L \subset W$ regular at $p_{\infty}^{ \pm}$. They vanish at four points

$$
p_{\alpha}^{\prime}=\left(V\left(z_{\alpha}^{\prime}\right), z_{\alpha}^{\prime}\right) \quad \text { or } \quad p_{\alpha}^{\prime \prime}=\left(-V\left(z_{\alpha}^{\prime \prime}\right), z_{\alpha}^{\prime \prime}\right),
$$

respectively, where $z_{\alpha}^{\prime}$ are the roots of $U$ and $z_{\alpha}^{\prime \prime}$ are those of $W$. Hence the degree of the line bundle $L$ is equal to $4 . H^{0}(L)$ has dimension 2 and is spanned by the two solutions (5.55).

\subsection{Angle variables}

The knowledge of the bundle $L$ may be encoded in the image $w$ of $L$ in the Jacobian $J^{4}(\mathcal{S})$ under the Abel map:

$$
w=\sum_{\alpha=1}^{4} \int_{p_{0}}^{p_{\alpha}^{\prime}} \Omega=\sum_{\alpha=1}^{4} \int_{p_{0}}^{p_{\alpha}^{\prime \prime}} \Omega
$$

were $\Omega=\left(\Omega^{b}\right)$ is the vector of the abelian differentials (5.49) on $\mathcal{S}$ and $p_{0}$ is a fixed point of $\mathcal{S}$. Under the infinitesimal time evolution (5.45) inducing the changes $\delta_{n} U, \delta_{n} V, \delta_{n} W$, of the polynomials $U, V, W$, the image of the Abel map changes by

$$
\delta_{n} w^{b}=\sum_{\alpha=1}^{4} \frac{z_{\alpha}^{\prime b} \delta_{n} z_{\alpha}^{\prime}}{V\left(z_{\alpha}^{\prime}\right)}=-\sum_{\alpha=1}^{4} \frac{z_{\alpha}^{\prime \prime b} \delta_{n} z_{\alpha}^{\prime \prime}}{V\left(z_{\alpha}^{\prime \prime}\right)} .
$$

The variations of the zeros of $U$ are

$$
\delta_{n} z_{\alpha}^{\prime}=-\frac{\delta_{n} U\left(z_{\alpha}^{\prime}\right)}{U^{\prime}\left(z_{\alpha}^{\prime}\right)}
$$

and similarly for $\delta_{n} z_{\alpha}^{\prime \prime}$. A direct calculation gives

$$
\delta_{n} U(z)=\left\{h_{n}, U(z)\right\} \delta t_{n}=4 i \prod_{m \neq n}\left(z_{n}-z_{m}\right)^{-1} \frac{V\left(z_{n}\right) U(z)-U\left(z_{n}\right) V(z)}{z-z_{n}} \delta t_{n},
$$


see [19], page 3.69. Hence

$$
\delta_{n} w^{b}=4 i \prod_{m \neq n}\left(z_{n}-z_{m}\right)^{-1} U\left(z_{n}\right) \delta t_{n} \sum_{\alpha=1}^{4} \frac{z_{\alpha}^{\prime b}}{\left(z_{\alpha}^{\prime}-z_{n}\right) U^{\prime}\left(z_{\alpha}^{\prime}\right)} .
$$

The vanishing of the sum of residues of the meromorphic form $\frac{z^{b} d z}{\left(z-z_{n}\right) U(z)}$ implies that the last sum is equal to $-z_{n}^{b} U\left(z_{n}\right)^{-1}$ so that

$$
\delta w^{b}=\left\{w^{b}, h_{n}\right\} \delta t_{n}=\frac{4}{i} \prod_{m \neq n}\left(z_{n}-z_{m}\right)^{-1} z_{n}^{b} \delta t_{n}
$$

which does not depend on the phase-space variables. We infer that the Hamiltonians $h_{n}$ generate constant flows on the complex torus $J^{4}(\mathcal{S}) \cong \mathbb{C}^{3} / \Lambda$ where $\Lambda$ is the lattice of periods of $\Omega$. Modulo a constant linear transformation, the coordinates $w^{b}, b=0,1,2$, provide together with 3 of the Hamiltonians $h_{n}$ a Darboux coordinate system for $T^{*} \mathbb{P}^{3}$. We have thus found the angle variables of the Hitchin system (as the angles of $J^{4}(\mathcal{S})$ ).

It should be stressed that the above approach based on the Lax matrix is simpler then the one obtained by following the general procedure for the Hitchin systems. In particular, Eq. (1.7) giving the spectral curve $\mathcal{C}$ in the general approach is, as shown in [13],

$$
\xi^{2}=-4 \prod_{n=1}^{6}\left(z-z_{n}\right) \sum_{\substack{n, m=1 \\ n \neq m}}^{6} \frac{J_{n m}^{2}}{\left(z-z_{n}\right)\left(z-z_{m}\right)}
$$

to which one has to add the equation (5.2) of the original curve $\Sigma$ of genus $2 . \mathcal{C}$ is of genus 5

and it is a ramified cover of both $\Sigma$ (by forgetting $\xi$ ) and $\mathcal{S}$ (by setting $\sigma=\frac{i}{2 \sqrt{2}} \xi y$ ). While the general construction would give the angle variables as those of a 3-dimensional Prym variety in the 5 -dimensional Jacobian of degree -2 line bundles on $\mathcal{C}$, the Lax approach gave them as the angles of the degree 4 Jacobian of $\mathcal{S}$.

\section{$5.9 \quad$ KZB connection}

The determinant bundle $\mathcal{L}$ over the moduli space $\mathcal{N}_{s s} \cong \mathbb{P} \Theta_{2}$ of the holomorphic $S L_{2^{-}}$ bundles over the genus 2 curve coincides with the dual of the tautological bundle on $\mathbb{P} \Theta_{2}$ so that $H^{0}\left(\mathcal{L}^{k}\right)$ is the space of homogeneous polynomials $\Psi$ of degree $k$ on the space $\Theta_{2}$. The Lie algebra $s_{6}$ acts in the space $\Theta_{2}$ by the first order differential operators, still denoted by $J_{n m}$, satisfying the commutation relations (5.34), (5.35) with the Poisson bracket replaced by the commutator. In the $(p, q)$-language they are obtained by replacing $p_{n}$ 's in the expressions (5.36) for $J_{n m}$ by $-\partial_{x_{n}}$. The KZB connection for the case in question has been work out in [15]. It takes the form (up to a scalar 1-form)

$$
\begin{aligned}
\nabla_{\delta \mu}^{\mathrm{KZB}} \Psi & =\sum_{n=1}^{6} \delta z_{n}\left(\partial_{z_{n}}-\frac{1}{\kappa} H_{n}\right) \Psi \\
\nabla_{\delta \mu}^{\mathrm{KZB}} \Psi & =\sum_{n} \overline{\delta z}_{n} \partial_{\bar{z}_{n}} \Psi
\end{aligned}
$$


where

$$
H_{n}=-\frac{1}{2} \sum_{m \neq n}^{6} \frac{J_{n m}^{2}}{z_{n}-z_{m}}
$$

so that $\frac{1}{k} H_{n}$ is a quantization of $\frac{2 k}{(2 \pi i)^{2}} h_{n}$ obtained by the replacement $J_{n m} \mapsto \frac{2 \pi}{k i} J_{n m}$ in the classical expression for $h_{n}$. The quantum Hamiltonians $H_{n}, n=1, \ldots, 6$, are commuting second order differential operators on $\Theta_{2} \cong \mathbb{C}^{4}$.

\section{Conclusions}

We have described above in explicit terms the Hitchin integrable systems and the KnizhnikZamolodchikov-Bernard connection in the genus 0, 1 and 2 geometries, the last case only for $G=S L_{2}$ and with no punctures. The main original contribution of the paper is the construction of the angle variables of the genus 2 system. It is a modification of a similar construction, based on the use of a Lax matrix, for the classical Neumann system. The diagonalization of the quantized Hamiltonians $H_{n}$ entering the genus 2 KZB connection for $G=S L_{2}$ as well as the identification of the genus 2 Hitchin systems with punctures and for different groups remain open problems.

\section{References}

[1] Avan, J., Talon, M.: Poisson Structure and Integrability of the Neumann-Moser-Uhlenbeck Model. Int. J. Mod. Phys. A 5, 4477-4488 (1990)

[2] Beilinson, A. A., Drinfeld, V. G. : Quantization of Hitchin's Fibration and Langlands Program. Preprint (1994)

[3] Bernard, D.: On the Wess-Zumino-Witten Models on the Torus. Nucl. Phys. B 303, 77-93 (1988)

[4] Bernard, D.: On the Wess-Zumino Model on Riemann Surfaces. Nucl. Phys. B 309, 145-174 (1988)

[5] Enriquez, B. Rubtsov, V.: Hitchin Systems, Higher Gaudin Operators and r-Matrices. Math. Res. Lett. 3, 343-357 (1996)

[6] Etingof, P., Kirillov, A., Jr.: Representations of Affine Lie Algebras, Parabolic Differential Equations and Lamé Functions. Duke Math. J. 74, 585-614 (1994)

[7] Falceto, F., Gawędzki, K.: Chern-Simons States at Genus One. Commun. Math. Phys. 159, 549-579 (1994)

[8] Falceto, F., Gawędzki, K.: Unitarity of the Knizhnik-Zamolodchikov-Bernard Connection and the Bethe Ansatz for the Elliptic Hitchin Systems. Commun. Math. Phys. 183, 267-290 (1997)

[9] Felder, G., Varchenko, A.: Integral Representations of Solutions of the Elliptic KnizhnikZamolodchikov-Bernard Equations. Int. Math. Res. Not. 5, 221-233 (1995)

[10] Felder, G., Wieczerkowski, C.: Conformal Blocks on Elliptic Curves and the KnizhnikZamolodchikov-Bernard Equations. Commun. Math. Phys. 176, 113-161 (1996)

[11] Frenkel, E.: Affine Algebras, Langlands Duality and Bethe Ansatz. In: XI ${ }^{\text {th }}$ International Congress of Mathematical Physics, ed. Iagolnitzer, D., International Press, Boston 1995, pp. 606-642

[12] Gawȩdzki, K.: Constructive Conformal Field Theory. In: Functional Integration, Geometry and Strings, eds. Haba, Z., Sobczyk, J., Birkhäuser, Basel, Boston Berlin 1989, p.. 277-302 
[13] Gawȩdzki, K., Tran-Ngoc-Bich, P.: Self-Duality of the $S L_{2}$ Hitchin Integrable System at Genus Two. solv-int/9710025, to appear in Commun. Math. Phys.

[14] van Geemen, B., Previato, E.: On the Hitchin System. Duke Math. J. 85, 659-683 (1996)

[15] van Geemen, B., de Jong, A. J.: On Hitchin's Connection. alg-geom/9701007

[16] Hitchin, N. J.: Stable Bundles and Integrable Systems. Duke Math. J. 54, 91-114 (1987)

[17] Hitchin, N. J.: Flat Connections and Geometric Quantization. Commun. Math. Phys. 131, 347-380 (1990)

[18] Knizhnik, V. G., Zamolodchikov, A. B.: Current Algebra and Wess-Zumino Model in Two Dimensions. Nucl. Phys B 247, 83-103 (1984)

[19] Mumford, D.: Tata Lectures on Theta II. Birkhäuser, Basel 1984

[20] Markman, E.: Spectral Curves and Integrable Systems. Comp. Math. 93, 255-290 (1994)

[21] Narasimhan, M. S., Ramanan, S.: Moduli of Vector Bundles on a Compact Riemann Surface. Ann. Math. 89, 19-51 (1969)

[22] Nekrasov, N.: Holomorphic Bundles and Many-Body Systems. Commun. Math. Phys. 180, 587-603 (1996)

[23] Neumann, C.: De Problemate quodam Mechanico, quod ad Primam Integralium Ultraellipticorum Classem Revocatur. Crelle 56 (1859)

[24] Verlinde, E: Fusion Rules and Modular Transformations in 2D Conformal Field Theory. Nucl. Phys. B 300 [FS 22], 360-376 (1988)

[25] Witten, E: Quantum Field Theory and Jones Polynomial. Commun. Math. Phys. 121, 351$399(1989)$ 